\title{
Diseño y validación de una escala para evaluar el Riesgo Suicida (ERS) en adolescentes colombianos*
}

\section{Design and Validation: A scale for the Risk of Suicide (ERS) in Colombian Adolescents}

\author{
Marly Johana Bahamón Muñetón ${ }^{\mathrm{a}}$ \\ Universidad Simón Bolívar, Colombia \\ ORCID: http://orcid.org/0000-0003-2528-994X \\ Yolima Alarcón-VÁsquez \\ Universidad Simón Bolívar, Colombia \\ ORCID: http://orcid.org/0000-0001-6357-8908
}

a Autor de correspondencia. Correo electrónico: mbahamon@unisimonbolivar.edu.co

Para citar este artículo: Bahamón Muñetón, M. J., \& Alarcón-Vásquez, Y. (2018). Diseño y validación de una escala para evaluar el riesgo suicida (ERS) en adolescentes colombianos. Universitas Psychologica, 17(1), 1-15. https://doi.org/10.11144/Javeriana.upsy 17-4.dvee

\section{RESUMEN}

El objetivo del estudio fue construir y validar una escala para la evaluación del riesgo suicida en adolescentes. Los ítems iniciales se elaboraron con base en la revisión bibliográfica y se sometió a evaluación de expertos con el fin de analizar aspectos teóricos y lingüísticos. La muestra fue de 537 adolescentes entre 13 y 18 años, con una media de 15.2 ( $D E=1.1), 268$ hombres (49.9\%) y $269(50.1 \%)$ mujeres. La consistencia interna total del instrumento fue de 0.934 ; las escalas mostraron alfa de Cronbach entre 0.71 y 0.929 ; se aplicó el omega de Mcdonald con el uso del software $\mathrm{R}$ y los resultados fueron muy similares. Se realizó análisis factorial exploratorio método VARIMAX y análisis factorial confirmatorio con el uso del programa estadístico AMOS. Los análisis de ítems, correlación y efectos de suelo y techo mostraron correlaciones entre $0.411 * *$ y $0.784 * *$ con el puntaje global. Las correlaciones de los ítems con las dimensiones fluctuaron entre $0.71 * *$ y $0.908 * *$. Una vez obtenida la agrupación de los ítems en cuatro factores, se hallaron correlaciones significativamente altas entre las subescalas con el puntaje global (0.749**a 0.868**). En conclusión, el instrumento constituye una medida válida y confiable para la evaluación del riesgo suicida en adolescentes colombianos.

Palabras clave

riesgo suicida; adolescencia; suicidio; evaluación suicidio; escala suicidio; ERS.

\begin{abstract}
This study aims to construct and validate a scale for the assessment of suicide risk in adolescents. Initial items were prepared based on bibliographical review and were subject to expert evaluation with the objective of analyzing theoretical and linguistic aspects. 537 adolescents between 13 and 18 years old with a mean of $15.2(\mathrm{SD}=1.1)$, 268 being male (49.9\%) and 269 (50.1\%) being womenfemale were sampled. The internal consistency of the instrument was .934 , the scales showed Cronbach's alphas between 0.71 and 0.929, it was applied from Mcdonald's Omega with the use of software $\mathrm{R}$ and the results were very similar. Exploratory factorial analysis was made VARIMAX method and confirmatory factorial analysis was determined with the use of the
\end{abstract}


statistical program masters. The items analysis, correlation, and effects of floor and ceiling, and showed correlations between $0.411 * *$ and $0.784 * *$ with a global score. The items correlations fluctuated between 0.71 and $0.908 * * * *$. In conclusion, the instrument shows to be a valid and reliable measure for suicide risk in an adolescent assessment tool.

Keywords

risk suicide; adolescence; suicide; suicide scale; ERS.

A nivel mundial, el suicidio se considera como la segunda o tercera causa de muerte en jóvenes entre los 15 y 19 años de edad (Larraguibel, González, Martínez, \& Valenzuela, 2000). En Colombia, según reportes oficiales, el suicidio es la cuarta forma de violencia en el país y del porcentaje total, el $10.5 \%$ corresponde a jóvenes entre los 15 y 18 años (Ramírez \& Naranjo, 2014).

Los aportes teóricos actuales sobre el suicidio proponen la existencia de múltiples aspectos que interaccionan en la configuración del fenómeno (Legido, 2012). De hecho, existen diferentes modelos que intentan abordar la conducta suicida; uno de ellos hace alusión a los modelos "multidimensionales" que procuran analizar el fenómeno, articulando diferentes perspectivas; dentro de los más reconocidos se encuentran: el modelo de trayectorias de desarrollo del suicidio de Silverman y Felner (1995), el modelo basado en el estado de la mente de Bonner y Rich (1987), el modelo cúbico del suicidio de Shneidman (1992) y el modelo de sobreposición de Blumenthal (1988).

Por su parte, el riesgo suicida es considerado como un conjunto de acciones y cogniciones que se configuran en el sujeto, quien a través de pensamientos o comportamientos busca la propia muerte (Wolfersdorf \& Kaschka, 1995). También se ha conceptualizado como la capacidad de predicción con base en la historia pasada y actual del sujeto, para determinar su potencial autodestructivo (González-Forteza \& AndradePalos, 1995). En relación con el riesgo suicida, se han estudiado factores asociados tales como la depresión, la desesperanza, ideación, planeación e intencionalidad suicida, aislamiento y familia, en su condición de aspectos que potencian la posibilidad de suicidio en la adolescencia, configurando en el individuo un patrón que favorece la aparición del suicidio en este grupo poblacional. A continuación, se exponen los aspectos mencionados, sin embargo, estos son analizados de manera independiente en los estudios.

A partir de lo anterior, el presente estudio tuvo por objetivo construir y validar una escala para la evaluación del riesgo suicida en adolescentes. Esta investigación se centra en un abordaje psicosocial del fenómeno en adolescentes y, por tanto, involucra elementos de orden individual y social (Gould, Fisher, Parides, Flory, \& Shaffer, 1996). La evaluación del riesgo suicida, desde esta propuesta, busca indagar por aspectos que aumentan la probabilidad del suicidio en adolescentes.

\section{Depresión y desesperanza}

En relación con el riesgo suicida, la depresión y la desesperanza se han considerado como variables intervinientes en la aparición y mantenimiento del fenómeno; al respecto, existen varios estudios que documentan la existencia de una relación significativa entre depresión y conducta suicida (Cova, Rincón, \& Melipillán, 2007; Fleming, Merry, Robinson, Denny, \& Watson 2007; Ortin, Lake, Kleinman, \& Gould, 2012; World Health Organization [WHO], 2008; Shaffer et al., 2004; Shaffer, Fisher et al., 1996; Shaffer, Gould et al., 1996). Uno de los trastornos con mayor prevalencia en personas que muestran riesgo suicida alto es la depresión, tanto que el suicidio se ha considerado como la complicación más grave de la depresión (Gómez et al., 2013).

Así mismo se ha definido que uno de los rasgos propios de personas con riesgo suicida es la desesperanza elevada. La desesperanza hace alusión a un conjunto de expectativas negativas hacia el futuro, y se considera como un factor relevante en el estudio del riesgo suicida, teniendo en cuenta que es un componente fuerte de la depresión y se le reconoce como un elemento central en la predicción del suicidio (Gómez, Núñez, \& Lolas, 1992; Gómez et al., 
1998; Runeson, Tidelman, Dahlin, Lichtenstein, \& Langström, 2010; Souminen, Isometsa, \& Lönquist, 2004).

La desesperanza funciona como un estilo atribucional que favorece el desarrollo de inferencias negativas sobre las experiencias y sucesos vitales que experimenta el individuo, cuestión que la convierte en un factor cognitivo importante para la predicción del intento suicida. Varios estudios sobre el tema exponen que la desesperanza diferencia a las personas que intentan suicidarse una vez, de aquellos que repiten este suceso (Kamath, Reddy, \& Kandavel, 2007; Kuo, Gallo, \& Eaton, 2004), y que entre mayor es el nivel de desesperanza, mayor es la gravedad del intento (Soloff, Lynch, Kelly, Malone, \& Mann, 2000). Según Fawcett, Busch, Jacobs, Kravitz y Fogg (1997), el suicidio es resultado de un estado de desesperanza motivado por la necesidad de escapar a la realidad vivenciada.

\section{Ideación, planeación e intencionalidad suicida}

Los adolescentes con riesgo suicida elevado han considerado previamente la idea de quitarse la vida, se trata de un proceso en el cual, el sujeto progresivamente se va persuadiendo acerca de por qué quitarse la vida y cómo hacerlo. En este sentido, Beck, Kovacs y Weissman (1976) declararon que las ideas, los intentos y el suicidio forman parte de un continuo de suicidabilidad creciente. La presencia de ideación suicida se considera como un signo de alerta que pone en evidencia la vulnerabilidad de la persona al suicidio. Algunos investigadores señalan que las ideas suicidas sumadas a la planificación del suicidio aumentan significativamente la probabilidad de su ejecución (Borges, Angost, Nock, Ruscio, \& Kessler, 2006; Powell, Geddes, Deeks, Goldacre, \& Hawton, 2000).

Referente al intento suicida, se ha determinado que la mitad de los adolescentes que cometen suicidio ha tenido intentos previos (Cooper et al., 2005) y se cree que adolescentes con intento suicida presentan un riesgo suicida elevado, particularmente en los diez años siguientes, puesto que la mitad de quienes cometen suicidio lo han intentado previamente (Baader, Urra, Millán, \& Yáñez, 2011; Harriss, Hawton \& Zahl, 2005). Algunas investigaciones refieren que el riesgo de suicidio permanece durante varios años después del primer intento, pero este es mayor en los primeros doce meses posteriores al intento, así mismo, el método puede ser más letal en tentativas futuras (Baader, Urra, Millán, \& Yáñez, 2011; Isometsa, 2001; Owens, Horrocks, \& House, 2002; Sakinofsky, 2000; Zahl \& Hawton, 2004).

\section{Aislamiento/Soporte social}

El apoyo social percibido por el sujeto se considera como un factor importante que puede activarse como riesgo o como factor protector. Si las cogniciones están referidas a ausencia de ese apoyo y cohesión, se determinará un factor importante de riesgo, en tanto si las interpretaciones de la persona enfocan hacia la contribución de apoyo y fuentes detectables de soporte, se constituirá en un factor protector (Gómez, Barrera, Jaar, Lolas, \& Núñez, 1995; Salazar \& González, 2017). Así, el aislamiento y las interpretaciones personales referidas a la ausencia de fuentes de apoyo o la ruptura de un lazo importante que se identificaba como proveedor afectivo, emocional o social, aumenta el riesgo suicida en adolescentes.

\section{Familia}

En relación con la familia, esta se considera como una estructura social que brinda soporte al niño y adolescente no solo para desarrollar procesos de socialización y aprendizaje, sino además para favorecer su desarrollo emocional y la consolidación de estrategias adecuadas que le permitan hacer frente a situaciones estresantes, resolver problemas cotidianos y sentirse seguros. Diferentes estudios, han hallado que la disfunción familiar en niños y adolescentes se constituye en factor de riesgo significativo para la depresión (Jiménez, Murgui, \& Musitu, 
2007) y el suicidio (Larraguibel et al., 2000; Sánchez-Loyo et al. 2014; Urzúa \& CaqueoUrízar, 2011). En este sentido, se ha identificado que los adolescentes que han realizado intentos suicidas provienen de familias más disfuncionales en comparación con aquellos que no lo intentan (Fortune \& Hawton, 2007; Kim \& Kim, 2008; Shur-Fen Gau et al., 2008; Silvikena \& Kvernmob, 2007). También se ha descubierto mayor presencia de ideación suicida en adolescentes de hogares desligados (Bahamón, Alarcón-Vásquez, García, \& Trejos, 2015; Pérez, Rodríguez, Dussán, \& Ayala, 2007; Sarmiento \& Aguilar, 2011). Gençöz y Or (2006) encontraron que la falta de sentido en conjunción con la desintegración familiar aumenta la probabilidad de suicidio. En tanto, otros estudios exponen que los adolescentes tienen mayores recursos protectores frente al suicidio, cuando sus padres atienden a sus necesidades emocionales y se comunican adecuadamente (Monge, Cubillas, Román, \& Abril, 2007; Oliva, 2006). Así mismo, la ausencia de calidez familiar, falta de comunicación con los padres y discordia familiar aparecen como los factores más frecuentemente asociados a la conducta suicida (Larraguibel et al., 2000).

$\mathrm{Al}$ parecer, las relaciones familiares son fundamentales para poblaciones latinas, tanto que se ha hallado que los problemas familiares se correlacionan con el intento suicida, independientemente de la presencia de trastornos mentales (Fortuna, Joy Perez, Canino, Sribney, \& Alegria, 2007).

\section{Evaluación del riesgo suicida}

Previamente, se han analizado algunas variables que con frecuencia se han asociado al riesgo suicida, aunque es necesario aclarar que este estudio ha procurado realizar una revisión no centrada en la patología o la presencia de trastornos mentales, puesto que se busca indagar por el riesgo suicida en fases iniciales para la detección temprana de algunos signos indicativos de mayor probabilidad de suicidio en el adolescente.
También es necesario indicar que son pocos los recursos con que se cuenta actualmente para la evaluación y detección temprana del riesgo suicida en población latina, y la mayoría de los existentes son instrumentos creados para otros contextos y traducidos al español con los problemas lingüísticos y comprensivos que esto genera (Agerbo, Nordentoft, \& Mortensen, 2002; Villacieros, Bermejo, Magaña, \& Fernández-Quijano, 2016).

Dentro de los instrumentos más reconocidos se encuentran: el Cuestionario de Indicadores de Riesgo CIR (Reyes \& Del Cueto de Inastrilla, 2003); las escalas de Beck (Beck, Schuyler, \& Hermann, 1974); la Escala de Riesgo Suicida de Plutchik (Plutchik, Van Praag, Conte, \& Picard, 1989); el Inventario Razones para Vivir (Linehan, Goodstein, Nielsen, \& Chiles, 1983); el CCCS-18 (Ruiz Hernández, NavarroRuiz, Torrente, \& Rodríguez, 2005); la escala de ideación suicida de Roberts validada para población mexicana por Rosales-Pérez, CórdovaOsnaya y Cortés-Granados (2015), los cuales fueron creados originalmente para población adulta.

En cuanto a instrumentos para la evaluación del riesgo suicida en adolescentes latinoamericanos, la lista es más recudida. Se puede contar con el Inventario de Orientaciones Suicidas ISO-30 de (King \& Kowalchuk, 1994, adaptación Fernández Liporace \& Casullo, 2006); el Inventario de riesgo suicida IRIS (Hernández-Cervantes \& Gómez-Maqueo, 2006), y en Colombia, el Inventario de Resiliencia ante el Suicidio (SRI-25) validado por Villalobos-Galvis, Arévalo y Rojas (2012); el inventario de razones para vivir (García et al., 2009) y el Inventario de Ideación Suicida Positiva y Negativa (PANSI) de Villalobos-Galvis, 2009.

Las evaluaciones con que se dispone actualmente, evalúan aspectos el riesgo desde perspectivas específicas que dan prevalencia a aspectos individuales que disponen al suicidio. El enfoque psicosocial desde el cual se definieron los factores que se indagarían en el instrumento propuesto, incorpora la ideación y depresión $y$, concebidos como elementos individuales, la ideación, planeación y autolesión. A su vez, 
el aislamiento/soporte social y falta de apoyo familiar como factores de riesgo social.

Teniendo en cuenta que son diferentes las estrategias de evaluación de la suicidabilidad, los instrumentos psicométricos ofrecen ventajas al momento de realizar procesos de screnning o tamizaje que pueden usarse en contextos clínicos, hospitalarios y educativos. Así, la escasez de instrumentos contextualizados para población adolescente muestra una dificultad que debe ser atendida.

\section{Método}

\section{Participantes y procedimiento}

La muestra estuvo conformada por 537 adolescentes entre 13 y 18 años con una media de $15.2(D E=1.1)$ de edad que accedieron voluntariamente a participar del estudio, firmando su asentimiento y entregando a los investigadores el consentimiento informado de sus padres o quien hiciera las veces de su representante legal. El muestreo fue no probabilístico; el procedimiento para la detección de los sujetos se realizó en instituciones educativas públicas de la ciudad de Barranquilla y su área metropolitana, seleccionando un total de cinco instituciones. Se consideró como criterio de inclusión el ser colombiano, adolescente y estar escolarizado. El instrumento fue cumplimentado por 268 hombres (49.9 \%) y 269 (50.1\%) mujeres.

\section{Instrumentos}

Escala para la evaluación del riesgo suicida (ERS)

Se trata de una escala tipo Likert, diseñada por las autoras y compuesta por 20 ítems con seis opciones de respuesta donde 1 es totalmente en desacuerdo, 2 = en desacuerdo, 3 $=$ parcialmente en desacuerdo, $4=$ parcialmente de acuerdo, 5 = de acuerdo, $6=$ totalmente de acuerdo. Los ítems se refieren a afirmaciones pensando en lo ocurrido en los últimos seis meses.
Evalúa los siguientes factores: 1) Depresión y desesperanza, 2) Ideación, planeación y autolesión, 3) Aislamento/Soporte social, 4) Falta de apoyo familiar (Apéndice 1).

Inventario de riesgo suicida IRIS de HernándezCervantes y Gómez-Maqueo (2006)

El IRIS consta de 50 reactivos con afirmaciones de cinco opciones de respuesta: a) Todo este tiempo, b) Casi siempre, c) Algunas veces, d) Rara vez y e) No, vigentes dentro de los últimos seis meses de vida del adolescente. Desarrollado con base en el modelo teórico de suicidio en niños y adolescentes de Shaffer (Shaffer, Gould, \& Hicks, 1994; Shaffer, Gould et al., 1996; Shaffer \& Greenberg, 2002), tiene como propósito identificar adolescentes con alto, medio o bajo riesgo suicida, considerando la severidad de su sistema de creencias. Las áreas que evalúa el instrumento son: ideación suicida, satisfacción en la vida, dificultades interpersonales, dificultades en la escuela, signos de alerta, planeación e intención suicida, malestar personal psicológico y desesperanza. Esta prueba fue validada en población mexicana, y para efectos de este estudio se analizó el índice de confiabilidad $(\alpha$ $=0.976)$ y análisis factorial confirmatorio que permitió identificar un $\chi 2=4248.93$; CFI $=0.91$ y RMSEA 0.06. Los datos expuestos permitieron determinar que se trataba de una prueba válida confiable para esta población.

Symptom Check List 90 (SLC-90)

Traducida y adaptada por Cruz, López, Blas, González y Chávez (2005), se trata de una escala compuesta por 90 ítems agrupados en nueve dimensiones clínicas primarias: Somatización, Obsesiones/ Compulsiones, Sensibilidad interpersonal, Depresión, Ansiedad, Hostilidad, Ansiedad fóbica, Ideación paranoide, Psicoticismo y tres índices globales: Índice de Severidad o Gravedad Global (ISG), Total de Síntomas Positivos (TSP) e Índice de Malestar Positivo (IMP). De manera específica para este estudio, se analizó solamente 
la información referida a presencia de síntomas depresivos. Este instrumento fue validado en población argentina, y para efectos del presente estudio se analizó la confiabilidad de la escala utilizada (depresión), la cual arrojó un $\alpha=0.879$.

\section{Análisis estadísticos}

Para la evaluación de la consistencia interna se utilizaron el alfa de Cronbach y el Omega de McDonald. Seguidamente, se realizaron análisis factoriales de tipo exploratorio a través del método de componentes principales con rotación VARIMAX. Se analizaron las correlaciones entre las dimensiones con la escala global mediante el coeficiente de correlación de Pearson. Finalmente, se llevó a cabo análisis factorial confirmatorio.

\section{Consideraciones éticas}

Teniendo en cuenta que los participantes del estudio en su mayoría eran menores de edad, se procedió a solicitar la autorización de las autoridades escolares para la recolección de la información. Se citó a los padres de familia para otorgar información sobre la participación de sus hijos en el estudio, se solicitó firma de consentimiento informado al representante legal de cada adolescente. El modo de aplicación fue grupal; se brindó la información sobre confidencialidad y uso de la información y se pidió asentimiento a los participantes para confirmar la voluntariedad de su participación en el estudio.

\section{Resultados}

\section{Consistencia interna}

El análisis de consistencia interna mediante el alfa de Cronbach para la totalidad de los ítems fue de 0.934, es decir, el instrumento en su conjunto mostró valores de confiabilidad excelente. De igual manera, el análisis de confiabilidad de las subescalas arrojó valores entre 0.71 y 0.929 los cuales son considerados muy buenos (Tabla 1). Adicionalmente, se realizó análisis de confiabilidad mediante el Omega de McDonald, el cual mostró resultados muy similares a los presentados mediante el análisis de confiabilidad de Cronbach.

\section{Tabla 1}

Estadísticos descriptivos de las escalas de evaluación del riesgo suicida ERS 20 items

\begin{tabular}{lrrrrrr}
\hline \multicolumn{1}{c}{ Escalas } & Mín. & Máx. & Media & DE & \multicolumn{1}{c}{$\alpha$} & $\begin{array}{c}\text { Omega } \\
\text { McDonald }\end{array}$ \\
\hline Depresión y desesperanza & 6 & 36 & 10.27 & 6.12 & 0.903 & 0.903 \\
Ideación, planeación y autolesión & 6 & 36 & 11.05 & 7.37 & 0.929 & 0.929 \\
Aislamiento & 4 & 24 & 10.8 & 5.18 & 0.71 & 0.711 \\
Falta de apoyo familiar & 4 & 24 & 8.06 & 4.88 & 0.839 & 0.84 \\
Escala completa ERS & 20 & 119 & 40.18 & 19.56 & 0.934 & 0.933 \\
\hline
\end{tabular}

\section{Análisis de ítems}

En la Tabla 2, se presentan las correlaciones obtenidas para cada ítem en relación con su factor y con el puntaje total obtenido en la escala, así como la distribución porcentual de cada opción de respuesta.

En general, las correlaciones del ítem con su dimensión factor oscilaron entre $0.71 * *$ y $0.908^{* *}$; en relación con los factores del instrumento, los datos fueron los siguientes: Depresión y desesperanza entre 0.599** y $0.745^{* *}$; Ideación, planeación y autolesión entre $0.693 * *$ y $0.784 * *$; Aislamiento/Apoyo social entre $0.411 * *$ y $0.69 * *$ y Falta de apoyo familiar entre $0.592 * *$ y $0.731 * *$. Se observa que la distribución de las respuestas tiende a centrarse en los puntajes bajos. 
Tabla 2

Análisis de items: correlación y efectos suelo y techo

\begin{tabular}{|c|c|c|c|c|c|c|c|c|}
\hline \multirow[t]{2}{*}{ Ítem } & \multicolumn{2}{|c|}{ Correlación ítem } & \multicolumn{6}{|c|}{$\begin{array}{c}\text { Distribución porcentual por } \\
\text { respuesta }\end{array}$} \\
\hline & Dim. & Global & 1 & 2 & 3 & 4 & 5 & 6 \\
\hline Creo que es mejor no hacer planes para un futuro. & $0.739 * *$ & $0.599 * *$ & 69.3 & 9.9 & 11.5 & 3.2 & 2.4 & 3.7 \\
\hline Pienso que el futuro es incierto y tri & $0.834 * *$ & $0.732^{* * *}$ & 69.3 & 10.8 & 11.4 & 3.4 & 1.7 & 3.5 \\
\hline 3. Creo que nada bueno me espera & $0.852^{* * *}$ & $0.72^{* * *}$ & 68.7 & 10.1 & 11.4 & 4.8 & 3 & \\
\hline 4. Pienso que la vida no tiene cosas buenas para ofrecerme. & $0.866^{* *}$ & $0.737 * *$ & 70.9 & 9.3 & 12.1 & 4.1 & 1.5 & \\
\hline $\begin{array}{l}\text { 5. Mi vida ha sido un fracaso y dudo que cambie en un } \\
\text { futuro. }\end{array}$ & $0.836^{* * *}$ & $0.745^{* * *}$ & 69.3 & 11.5 & 11.9 & 3.4 & 1.9 & \\
\hline $\begin{array}{l}\text { 6. Me siento incómodo(a) conmigo mismo(a) y con lo que } \\
\text { he logrado. }\end{array}$ & $0.816^{* * *}$ & $0.732^{* * *}$ & 59.8 & 12.3 & 13 & 7.4 & 4.1 & 3.4 \\
\hline 7. He pensado seriamente en quitarme la vida. & $0.855^{* * *}$ & $0.759 * *$ & 66.3 & 6.5 & 9.1 & 9.9 & 4.3 & 3.9 \\
\hline $\begin{array}{l}\text { 8. Pienso que la muerte es la única manera de terminar con } \\
\text { mi sufrimiento. }\end{array}$ & $0.811^{* *}$ & $0.762^{* *}$ & 72.1 & 6.5 & 12.3 & 4.3 & 1.7 & 3.2 \\
\hline 9. He planeado como quitarme la vida. & $0.908^{* *}$ & $0.784^{* *}$ & 67.8 & 6 & 11 & 7.3 & 5.6 & 2.4 \\
\hline 10. He intentado quitarme la vida a & $0.907^{* * *}$ & & 73.7 & 3.9 & 9.3 & 5.2 & 4.7 & 3.2 \\
\hline 11. He hecho cosas contra mí & & $0.749^{* * *}$ & 72.3 & 6 & 9.5 & 3.9 & 4.5 & 3.9 \\
\hline $\begin{array}{l}\text { 12. Me he lastimadoo herido inte- } \\
\text { 13. Me siento solo(a) en la vida. }\end{array}$ & $\begin{array}{c}0.799^{* *} \\
0.71^{* *}\end{array}$ & $\begin{array}{l}0.693^{* * *} \\
0.6{ }^{* *}\end{array}$ & $\begin{array}{l}59.4 \\
59.4\end{array}$ & $\begin{array}{r}9.1 \\
11.5\end{array}$ & $\begin{array}{r}8.9 \\
12.1\end{array}$ & $\begin{array}{l}7.8 \\
7.6\end{array}$ & $\begin{array}{l}7.6 \\
3.4\end{array}$ & $\begin{array}{l}7.1 \\
6\end{array}$ \\
\hline 14. Me es dificil confiar en otras personas. & $0.738^{* *}$ & $0.523 * *$ & 36.1 & 11.2 & 12.5 & 14.3 & 13.8 & 12.1 \\
\hline 15. Me gustaría tener amigos a quienes acudir cuando tengo & $0.722^{* * *}$ & $0.411^{* * *}$ & 31.8 & 6.9 & 10.2 & 12.1 & 17.52 & 21.4 \\
\hline $\begin{array}{l}\text { 16. Creo que las personas tienden a ignorarme. } \\
\text { 1. }\end{array}$ & $0.769^{* *}$ & $0.617^{* * *}$ & 49.3 & 11.2 & 15.1 & 6.5 & 8 & 9.9 \\
\hline 17. A mi familia le interesa poco lo que suceda conmigo. & $0.79^{* *}$ & $0.592^{* *}$ & 66.1 & 8.6 & 12.1 & 5 & 4.5 & 3.7 \\
\hline $\begin{array}{l}\text { 18. Prefiero acudir a personas diferentes a mi familia } \\
\text { cuando necesito ayuda. }\end{array}$ & $0.805^{* *}$ & $0.667^{* * *}$ & 45.8 & 13.2 & 12.8 & 11 & 9.5 & 7.6 \\
\hline $\begin{array}{l}\text { 19. Prefiero estar lejos de mi familia que compartir con } \\
\text { ellos. }\end{array}$ & $0.857^{* * *}$ & $0.731^{* * *}$ & 63.1 & 10.4 & 12.3 & 5.8 & 3.5 & 4.8 \\
\hline 20. Pienso que soy poco importante para mi familia. & $0.846^{* *}$ & $0.707^{* *}$ & 65 & 12.1 & 9.9 & 5.4 & 3.2 & 4.5 \\
\hline
\end{tabular}

Validez de constructo

Al realizar el análisis de correlación entre los factores de la escala de evaluación del riesgo suicida y entre cada factor y el puntaje total de la escala se evidencian correlaciones positivas significativas muy fuertes entre todos los elementos (Tabla 3).

\section{Tabla 3}

Correlaciones de Pearson entre factores y puntaje total

\begin{tabular}{lrrrrr}
\hline \multicolumn{1}{c}{ Factor } & Factor 1 & Factor 2 & Factor 3 & Factor 4 & Total ERS \\
\hline Factor 1. Depresión y desesperanza & 1 & $0.681^{* *}$ & $0.527^{* *}$ & $0.616^{* *}$ & $0.863^{* *}$ \\
$\begin{array}{l}\text { Factor 2. Ideación, planeación y } \\
\text { autolesión }\end{array}$ & $0.681^{* *}$ & 1 & $0.48^{* * *}$ & $0.606^{* *}$ & $0.868^{* *}$ \\
$\begin{array}{l}\text { Factor 3. Aislamiento/soporte social } \\
\text { Factor 4. Falta de apoyo familiar }\end{array}$ & $0.527^{* *}$ & $0.48^{* *}$ & 1 & $0.555^{* *}$ & $0.749^{* *}$ \\
\hline
\end{tabular}

Inicialmente, se construyeron 32 ítems para la evaluación del riesgo suicida en adolescentes, los cuales fueron evaluados por tres investigadores con experiencia en el tema para analizar la pertinencia en el contenido de los ítems y sus factores. Una vez identificada, se seleccionaron 20 ítems. Seguidamente, se comprobaron los supuestos del análisis factorial, obteniendo un índice de Kaiser Meyer Olkin cercano a 1 (0.946), la prueba de esfericidad de Bartlett significativa arrojó un chi cuadrado de 7197.604, $g l=190$ y un nivel de significancia de $<0.0001$. Se procedió a la ejecución del análisis factorial exploratorio mediante el método de extracción máxima verosimilitud y rotación VARIMAX. Los resultados de este procedimiento estadístico mostraron que la varianza se explicaba en un 69 $\%$ por cuatro factores. Los ítems se agruparon de acuerdo con lo propuesto teóricamente (Tabla 4).

Tabla 4

Matriz de factores rotados

\begin{tabular}{lrrrr}
\hline & \multicolumn{4}{c}{ Factor } \\
\cline { 2 - 5 } Ítem & $\begin{array}{c}\text { Depresión y } \\
\text { desesperanza }\end{array}$ & $\begin{array}{c}\text { Ideación, } \\
\text { planeación y } \\
\text { autolesión }\end{array}$ & $\begin{array}{c}\text { Aislamiento/ } \\
\text { Soporte } \\
\text { social }\end{array}$ & $\begin{array}{c}\text { Falta apoyo } \\
\text { familiar }\end{array}$ \\
\hline ERS4 & $\mathbf{0 . 7 9 5}$ & 0.253 & 0.181 & 0.168 \\
ERS3 & $\mathbf{0 . 7 3 4}$ & 0.219 & 0.215 & 0.208 \\
ERS5 & $\mathbf{0 . 6 9 3}$ & 0.283 & 0.268 & 0.178 \\
ERS2 & $\mathbf{0 . 6 7 2}$ & 0.321 & 0.173 & 0.211 \\
ERS6 & $\mathbf{0 . 6 4 2}$ & 0.259 & 0.222 & 0.292 \\
ERS1 & $\mathbf{0 . 5 6 8}$ & 0.235 & 0.197 & 0.071 \\
ERS10 & 0.263 & $\mathbf{0 . 8 6 8}$ & 0.207 & 0.123 \\
ERS11 & 0.259 & $\mathbf{0 . 8 4 7}$ & 0.202 & 0.14 \\
ERS9 & 0.327 & $\mathbf{0 . 7 5 3}$ & 0.245 & 0.189 \\
ERS7 & 0.339 & $\mathbf{0 . 6 6}$ & 0.206 & 0.251 \\
ERS12 & 0.228 & $\mathbf{0 . 5 9 5}$ & 0.208 & 0.285 \\
ERS8 & 0.538 & $\mathbf{0 . 5 6 5}$ & 0.202 & 0.133 \\
ERS20 & 0.295 & 0.225 & $\mathbf{0 . 7 3}$ & 0.211 \\
ERS17 & 0.162 & 0.23 & $\mathbf{0 . 6 6}$ & 0.147 \\
ERS19 & 0.335 & 0.246 & $\mathbf{0 . 6 4 2}$ & 0.27 \\
ERS18 & 0.262 & 0.234 & $\mathbf{0 . 4 9}$ & 0.332 \\
ERS16 & 0.224 & 0.166 & 0.234 & $\mathbf{0 . 6 2 2}$ \\
ERS14 & 0.154 & 0.185 & 0.081 & $\mathbf{0 . 5 7 2}$ \\
ERS15 & 0.065 & 0.079 & 0.129 & $\mathbf{0 . 4 7 7}$ \\
ERS13 & 0.419 & 0.13 & 0.365 & $\mathbf{0 . 4 6 9}$ \\
\hline & & & & \\
\hline
\end{tabular}

Finalmente, se ejecutó un análisis factorial confirmatorio para indagar por los índices de ajuste y bondad del instrumento que corroboraran su validez. Los resultados de éste análisis mostraron que la escala propuesta para la evaluación del riesgo suicida tiene un ajuste satisfactorio dado que el RMSEA es mayor que 0.06 y el CFI Y TLI presentaron valores mayores de 0.89 (Tabla 5).

Tabla 5

Análisis factorial confirmatorio ERS (Estimación con máxima verosimilitud)

\begin{tabular}{cccccccrc}
\hline Modelo & $\chi 2$ & gl & AIC & CFI & NNFI & RMR & RMSEA & TLI \\
\hline 20 ítems & 600.151 & 164 & 692.151 & 0.939 & 0.918 & 0.105 & 0.07 & 0.929 \\
\hline
\end{tabular}

\section{Validez convergente}

Teniendo en cuenta que en la bibliografía existente se hace alusión a la depresión como un factor relacionado con el riesgo suicida, se aplicó a los participantes el SLC-90 (subescala depresión), encontrando correlaciones significativas entre la subescala de 
depresión con todos los factores el ERS. Así mismo, los adolescentes cumplimentaron el IRIS, el cual se correlacionó significativamente con todos los factores del ERS y su puntaje total (Tabla 6).

Tabla 6

Correlaciones de Pearson del ERS y otros instrumentos

\begin{tabular}{lcc}
\hline \multicolumn{1}{c}{ Factores ERS } & IRIS & $\begin{array}{c}\text { SLC-90 } \\
\text { (depresión) }\end{array}$ \\
& $r$ & $r$ \\
\hline Factor 1.Depresión y desesperanza & $0.781^{* *}$ & $0.508^{* *}$ \\
Factor 2. Ideación e intencionalidad suicida & $0.759^{* *}$ & $0.511^{* *}$ \\
Factor 3. Aislamiento & $0.742^{* *}$ & $0.524^{* *}$ \\
Factor 4. Falta de apoyo familiar & $0.787^{* *}$ & $0.466^{* *}$ \\
\hline Total escala riesgo suicida ERS & $0.882^{* *}$ & $0.607^{* *}$ \\
\hline
\end{tabular}

** Correlación significativa

al 0.01 ( 2 colas). $N=537$

\section{Discusión de resultados}

Esta investigación tuvo como propósito construir y validar una escala para la evaluación del riesgo suicida en adolescentes, necesidad que surge de los escasos instrumentos contextualizados con que se cuenta para dicha evaluación y de los diferentes factores que intervienen en el fenómeno que pueden detectarse de manera temprana (Agerbo et al., 2002).

Los instrumentos para la detección de riesgo suicida en adolescentes con los que cuentan los profesionales en el área de la psicología han hecho énfasis en aspectos individuales del fenómeno, a excepción del IRIS (HernándezCervantes \& Gómez-Maqueo, 2006) que indaga por algunas condiciones contextuales referidas a la escuela. La escala propuesta retoma el contexto familiar como un factor de riesgo necesario de considerar, teniendo en cuenta las múltiples investigaciones que han hallado la relación entre algunas características del ambiente familiar y el potencial suicida en adolescentes (Larraguibel et al., 2000; SánchezLoyo et al., 2014; Urzúa \& Caqueo-Urízar, 2011).

Los cuatro factores que evalúa el instrumento propuesto han sido expuestos en la literatura como importantes en el riesgo suicida. El del Factor 1, Depresión y desesperanza, se define como la interacción entre la alteración del estado de ánimo conformada por diferentes patrones de comportamiento clínicamente significativos, caracterizada por una alteración del humor asociada al malestar personal (American Psychiatric Association, 1993, 2013) y la desesperanza hace referencia al conjunto de expectativas que se constituyen en un estilo atribucional, caracterizado por inferencias negativas acerca de las experiencias y los sucesos vitales así como expectativas negativas hacia el futuro (Kamath et al., 2007; Kuo et al., 2004; Runeson et al., 2010).

El Factor 2 hace alusión al continuo de suicidabilidad creciente en el adolescente y que involucra las ideas e intentos suicidas, es decir, pensamientos e ideas recurrentes acerca de querer quitarse la vida y las acciones o comportamientos encaminados a la consecución de esta meta (Borges et al., 2006; Gómez, 2012; Powell et al., 2000).

El Factor 3 se refiere a las interpretaciones del adolescente frente al contacto con otras personas y la apreciación subjetiva de tener poco contacto o relacionamiento con otras personas, ello incluye sentirse poco valorado por el contexto relacional significativo (Cacioppo \& Cacioppo, 2014; Cacioppo, Grippo, London, Goossens, \& Cacioppo, 2015; Ong, Uchino, \& Wethington, 2016). Si bien esta categoría se ha analizado en adultos mayores, surge la necesidad de explorarla a profundidad en adolescentes con riesgo suicida.

El Factor 4 guarda relación con las dificultades que percibe el adolescente en su contexto familiar y poco apoyo por parte de los miembros de la familia, que se traduce en poca atención a las necesidades emocionales del joven (Larraguibel et al., 2000; Monge et al., 2007; Oliva, 2006; Sánchez-Loyo et al., 2014; Urzúa \& CaqueoUrízar, 2011).

Un punto para analizar es la varianza explicada que fue de $69 \%$, por lo cual se hace necesario que en futuras investigaciones se contemplen otros elementos que pueden explicar el riesgo suicida para aumentar este porcentaje. Otro aspecto de consideración es la variabilidad del estrato socioeconómico en el que se ubicaron los participantes, puesto que todos correspondían 
a instituciones educativa públicas, lo cual implica poca representatitividad de estratos socioeconómicos altos; no obstante, algunas investigaciones han referido que las desventajas socioeconómicas muestran escasa evidencia de asociación al intento suicida (Baader et al., 2011).

La aplicación del instrumento propuesto puede realizarse en diferentes contextos y permite hacer una evaluación inicial sobre el riesgo suicida en adolescentes. En los Apéndices 2 y 3, se muestran los valores de los centiles por sexo que pueden utilizarse para realizar la interpretación de los puntajes obtenidos en cada subescala y en la escala global. La interpretación de los puntajes debe realizarse en su totalidad para valorar la probabilidad de riesgo, sin embargo, es necesario que se analice siempre de manera individual el Factor 2, correspondiente a Ideación, planeación y autolesión, independientemente del puntaje total, dado que los ítems indican riesgo directo.

Otro aspecto que se debe tener en cuenta se relaciona con la particularidad de este instrumento frente a los existentes para esta población, puesto que incorpora un enfoque psicosocial que valora no solo aspectos individuales o patológicos, sino además factores de riesgo ubicados en la esfera social en relación con el adolescente.

Por último, se concluye que la escala para la evaluación del riesgo suicida ERS mostró altos índices de confiabilidad; el análisis de validez de constructo mediante los índices del análisis confirmatorio demuestra que se trata de un instrumento útil, válido y confiable para evaluar el riesgo suicida en adolescentes colombianos.

\section{Referencias}

Agerbo, E., Nordentoft, M., \& Mortensen, P. (2002). Familial, psychiatric, and socioeconomic risk factors for suicide in young people: Nested case-control study. British Medical Journal, 325, 74-79. http://d x.doi.org/10.1136/bmj.325.7355.74
American Psychiatric Association. (1994). Diagnostic and statistical manual of mental disorders (4a. ed.). Washington, DC, EE. UU. : Autor.

American Psychiatric Association. (2013). Diagnostic and statistical manual of mental disorders (5a. ed.). Washington, DC, EE. UU. : Autor.

Baader, T., Urra, E., Millán, R., \& Yáñez, L. (2011). Algunas consideraciones sobre el intento de suicidio y su enfrentamiento. Revista Médica Clínica Las Condes, 22(3), 303-309. http://dx.doi.org/10.1016/S07168640(11) 70430-8

Bahamón, M., Alarcón-Vásquez, Y., García, C. \& Trejos, A. (2015). Riesgo suicida, funcionalidad familiar y esquemas maladaptativos en jóvenes universitarios. En Y. Alarcón-Vásquez, F. Vásquez, Y. Martínez \& W. Pineda (Eds.), Tendencias y avances en psicología. Aportes desde la investigación científica (pp. 197-222). Barranquilla, CO: Ediciones Universidad Simón Bolívar.

Beck, A., Kovacs, M., \& Weissman, A. (1976). Hopelessness and suicidal behavior. An overview. Journal of the American Medical Association, 234(11), 1146-1149. http://dx.doi.org/10.1001/jama .1975 .03260240050026

Beck, A. T., Schuyler, D., \& Hermann, I. (1974). Development of suicidal intent scales. En A. T. Beck, H. L. O. Resnick \& D. J. Lettieri (Eds.), The prediction of suicide (pp. 45-56). Bowie, MD: Charles Press.

Blumenthal, S. J. (1988). Suicide: A guide to risk factors, assessment, and treatment of suicidal patients. Medical Clinics of North América, 72 (4), 937-71. Recuperado de https://www.ncbi.nlm.nih.gov/pubmed/ 3287045

Bonner, R. L., \& Rich, A. R. (1987). Toward a predictive model of suicidal ideation and behavior: Some preliminary data in college students. Suicide and Life-Threatening Behavior, 17(1), 50-63. http://dx.doi.org/10 .1111/j.1943-278X.1987.tb00061.x 
Borges, G., Angost, J., Nock, M., Ruscio, A., $\&$ Kessler, R. (2006). A risk index for 12 month suicide attempts in the National Comorbidity Survey Replication (NCS-R). Psychological Medicine, 36, 1747-1757. http ://dx.doi.org/10.1017/S0033291706008786

Cacioppo, J., \& Cacioppo, S. (2014). Social relationships and health: The toxic effects of perceived social isolation. Social and Personality Psychology Compass, 8, 58-72. ht tp://dx.doi.org/10.1111/spc3.12087

Cacioppo, S., Grippo A., London, S., Goossens, L., \& Cacioppo J. (2015). Loneliness: Clinical import and interventions. Perspectives on Psychological Science, 10, 238-249. http://dx.doi.org/10.1177/174569 1615570616

Cooper, J., Kapur, N., Webb, R., Lawlor, M., Guthrie, E., Mackway-Jones, K., \& Appleby, L. (2005). Suicide alter deliberate self-ham: A 4 year cohort study. The American Journal of Psychiatry, 162(2), 297-303. http:// dx.doi.org/10.1176/appi.ajp.162.2.297

Cova, F., Rincón, P., \& Melipillán, R. (2007). Rumiación y presencia de sintomatología ansiosa y depresiva en adolescentes. Revista Mexicana de Psicología, 24, 175-183. Recuperado de http://www.redalyc.org/htm 1/2430/243020637003/

Cruz, F., López, B., Blas, G., González, M., \& Chávez, B. (2005). Datos sobre la validez y confiabilidad de la Symptom Check List 90 (SCL 90) en una muestra de sujetos mexicanos. Salud Mental, 28(1), 72-81. Recuperado de http://new.medigraphic.com/cgi-bin/res umen.cgi? IDARTICULO $=6689$

Fawcett, J., Busch, K., Jacobs, D., Kravitz, H., \& Fogg, L. (1997). Suicide a four pathway clinical-biochemical model. Annals of the New York Academy of Sciences, 836, 288-301. http://dx.doi.org/10.1111/j.1749. 6632.1997.tb52366.x

Fernández Liporace, M., \& Casullo, M. M. (2006). Validación factorial de una escala para evaluar riesgo suicida. Revista Iberoamericana de Evaluación Psicológica,
1(21), 9-22. Recuperado de http://www.red alyc.org/pdf/4596/459645448002.pdf

Fleming, T. M., Merry, S., Robinson, E., Denny, S., \& Watson, P. (2007). Self-reported suicide attempts and associated risk and protective factors among secondary school students in New Zealand. Australian and New Zealand Journal of Psychiatry, 41(3), 213-221. Recuperado de http://citeseerx.ist .psu.edu/viewdoc/download?doi=10.1.1.4 $58.2841 \&$ rep $=$ rep $1 \&$ type $=p d f$

Fortuna, L. R., Joy Perez, D., Canino, G., Sribney, W., \& Alegria, M. (2007). Prevalence and correlates of lifetime suicidal ideation and attempts among Latino subgroups in the United States. Journal of Clinical Psychiatry, 68(44), 572-581. Recuperado de https://www.ncbi.nlm.nih.gov/pmc/artic les/PMC2774123/

Fortune, S., \& Hawton, K. (2007). Suicide and deliberate self-harm in children and adolescents. Paediatrics and Child Health, 17, 443-447. http://dx.doi.org/10.1016/j.pa ed.2007.09.001

García, J., Palacio, C., Vargas, G., Arias, S., Ocampo, M., Aguirre, B.,... García, H. (2009). Validación del inventario de razones para vivir (RFL) en sujetos con conducta suicida de Colombia. Revista Colombiana de Psiquiatría, 38(1), 66-84. Recuperado de http://www.scielo.org.co/pd f/rcp/v38n1/v38n1a06.pdf

Gençöz, T., \& Or, P. (2006). Associated factors of suicide among university students: Importance of family environment. Contemporary Family Therapy, 28(2), 261-268. http://dx.doi.org/10.1007/s10591 $-006-9003-1$

Gómez, A. (2012). Evaluación del riesgo de suicidio: enfoque actualizado. Revista Médica Clínica. Condes, 23(5), 607-615.

Gómez, A., Barrera, A., Jaar, E., Lolas, F., \& Núñez, C. (1995). Apoyo social en el intento suicida. Psicopatología, 11: 54-58

Gómez, A., Núñez, C., \& Lolas, F. (1992). Ideación e intentos de suicidio en estudiantes de medicina. Revista de 
Psiquiatría de la Facultad Medicina Barcelona, 19(6), 265-272.

Gómez, A., Orellana, G., Jaar, E., Núñez, C., Montino, O., \& Lolas, F. (1998). La desesperanza como rasgo predictor del intento de suicidio. Psicopatología, 18, 113-116.

Gómez, C., Bohórquez, A., Gil, L. M., Jaramillo, L. E., García, J., Bravo, E., ... Palacio, C. (2013). Evaluación del riesgo de suicidio en la guía de práctica clínica para diagnóstico y manejo de la depresión en Colombia. Revista Colombiana de Psiquiatría, 43(1), 3-11. http://dx.doi.org/10.1016/j.rcp.2013. 11.001

González-Forteza, C., \& Andrade-Palos, P. (1995). La relación de los hijos con sus progenitores y sus recursos de apoyo: correlación de la sintomatología depresiva y la ideación suicida en los adolescentes mexicanos. Salud Mental, 18(4), 41-48. Recuperado de https://dialnet.unirioja.es/se rvlet/articulo? codigo $=5789289$

Gould, M., Fisher, P., Parides, M., Flory, M., \& Shaffer, D. (1996). Psychosocial risk factors of child and adolescent completed suicide. Archives of General Psychiatry, 53(12), 1155-1162. http://dx.doi.org/10.1001/arch psyc. 1996.01830120095016

Harriss, L., Hawton, K., \& Zahl, D. (2005). Value of measuring suicidal intent in the assessment of people attending hospital following self-poisoning or self-injury. The British Journal of Psychiatry, 186, 60-69. DOI: 10.1192/bjp.186.1.60

Hernández-Cervantes, Q. \& Gómez-Maqueo, E. (2006). Evaluación del riesgo suicida y estrés asociado en adolescentes estudiantes mexicanos. Revista Mexicana de Psicología, 23(1), 45-52

Isometsa, E. (2001). Psychological autopsy studies a review. European Psychiatry, 16(7), 379-85. DOI: http://dx.doi.org/10.1016/SO 924-9338(01)00594-6

Jiménez, T., Murgui, S., \& Musitu, G. (2007). Comunicación familiar y ánimo depresivo: el papel mediador de los recursos psicosociales del adolescente.
Revista mexicana de psicología, 24 (2), 259-271.

Kamath, P., Reddy, Y., \& Kandavel, T. (2007). Suicidal behavior in obsessive-compulsive disorder. Journal of Clinical Psychiatry, 68(11), 1741-50.

Kim, H. S. K., \& Kim, H. S. (2008). Risk factors for suicide attempts among Korean adolescents. Child Psychiatry Human Development, 39, 221-235. http://dx.doi.org /10.1007/s10578-007-0083-4

Kuo, W., Gallo, J., \& Eaton, W. (2004). Hopelessness, depression, substance disorder, and suicidality--a 13-year community-based study. Social Psychiatry and Psychiatric Epidemiology, 39(6), 497-50. DOI: $10.1007 / \mathrm{s} 00127-004-0775-z$

Larraguibel, M., González, P., Martínez, V., \& Valenzuela, R. (2000). Factores de riesgo de la conducta suicida en niños y adolescentes. Revista Chilena de Pediatría, 71(3), 183-191. http://dx.doi.org/10.4067/ S0370-41062000000300002

Legido, T. (2012). Clasificación de la conducta suicida utilizando cuestionarios psicométricos (Tesis doctoral inédita). Universidad de Alicante, España. Recuperado de http://dspace.uah.es/dspace/bitstream/h andle/10017/17103/TESIS_2012_TERES A_LEGIDO.pdf?sequence $=1$

Linehan, M., Goodstein, J., Nielsen, S., \& Chiles, J. (1983). Reasons for staying alive when you are thinking of killing yourself: The Reasons for Living Inventory. Journal of Consulting and Clinical Psychology, 51, 276-286. http://dx.doi.org/10.1037/0022-0 06X.51.2.276

Monge, J., Cubillas, M. J., Román, R., \& Abril, E. (2007). Intentos de suicidio en adolescentes de educación media superior y su relación con la familia. Psicología y Salud, 17, 45-51. Recuperado de http://revistas.uv.mx/index.php/psicysal ud/article/viewFile/738/1311

Oliva, A. (2006). Relaciones familiares y desarrollo adolescente. Anuario de Psicología, 37 (3), 209-223. Recuperado de h ttp://www.raco.cat/index.php/anuariopsico 
logia/article/viewFile/61838/82584\&q=ch icas

Ong, A., Uchino, B., \& Wethington, E. (2016). Loneliness and health in older adults: A mini-review and synthesis. Gerontology, 62, 443-449. http://dx.doi.org/10.1159/000441 651

Ortin, A., Lake, A., Kleinman, M., \& Gould, M. (2012). Sensation seeking as risk factor for suicidal ideation and suicide attempts in adolescence. Journal of Affective Disorders, 143, 214-222. http://dx.doi.org/10.1016/j.ja d.2012.05.058

Owens, D., Horrocks, J., \& House, A. (2002). Fatal and non-fatal repetition of self-harm. Systematic review. The British Journal of Psychiatry, 181, 193-199. http://dx.doi.org/ 10.1192/bjp.181.3.193

Pérez, I., Rodríguez, E., Dussán, M., \& Ayala, J. (2007). Caracterización psiquiátrica y social del intento suicida atendido en una clínica infantil, 2003-2005. Revista de Salud Pública, 9(2), 230-240. Recuperado de http://www.scielo.org.co/pdf/rsap/v9n2/ v9n2a07.pdf

Plutchik, R., Van Praag, H. M., Conte, H. R., \& Picard, S. (1989). Correlates of suicide and violent risk: The suicide risk measure. Comprehensive Psychiatry, 30, 1-7. http://dx .doi.org/10.1016/0010-440X(89)90053-9

Powell, J., Geddes, J., Deeks, J., Goldacre, M., \& Hawton, K. (2000). Suicide in psychiatric hospital in-patients: Risk factors and their predictive power. The British Journal of Psychiatry, 176, 266-272. http://dx.doi.org/ 10.1192/bjp.176.3.266

Ramírez, L., \& Naranjo, C. A. (2014). Comportamiento del suicidio. Colombia, 2014. Revista Forensis, 16(1), 319-350. Recuperado de http://www.medicinalegal.gov.co/docu ments/88730/1656998/Forensis + Interacti vo+2014.24-JULpdf.pdf/9085ad79-d2a9-4 c0d-a17b-f845ab96534b

Reyes, W. G., \& Del Cueto de Inastrilla, E. (2003). Factores psicosociales de riesgo de la conducta suicida. Revista Cubana de Medicina General Integral, 19(5).
Recuperado de http://scielo.sld.cu/scielo.ph p? script $=$ sci_arttext $\&$ pid $=$ S0864-212520 $03000500004 \& \operatorname{lng}=\mathrm{es} \& \operatorname{tlng}=\mathrm{es}$

Rosales-Pérez, J., Córdova-Osnaya, M., \& Cortés-Granados, R. (2015). Confiabilidad y validez de la escala de ideación suicida de Roberts. Journal of Behavior, Health Eु Social, 7(2), 31-41. http://dx.doi.org/10.5460/jbhsi .$v 7.2 .44302$

Ruiz Hernández, J., Navarro-Ruiz, J., Torrente, G., \& Rodríguez, A. (2005). Construcción de un cuestionario de creencias actitudinales sobre el comportamiento suicida: el CCCS-18. Psicothema, 17(4), 684-690. Recuperado de http://www.psicot hema.com/pdf/3166.pdf

Runeson, B., Tidelman, D., Dahlin, M., Lichtenstein, P., \& Langström, N. (2010). Method of attempted suicide as predictor of successful suicide: Natural long-term cohort study. BMJ, 341: c3222. http://dx.d oi.org/10.1136/bmj.c3222

Sakinofsky, I. (2000). Repetition of suicidal behaviour. En K. Hawton \& K. van Heeringen (Eds.), The international handbook of suicide and attempted suicide (pp. 385-404). Chichester, United Kingdom: Wiley.

Salazar, J. \& González, J. (2017). Relación entre riesgo suicida, autoestima, desesperanza y estilos de socialización parental en estudiantes de bachillerato. Psicogente, 20(37), 70-88. http://doi.org/10.17081/psic o.20.37.2419

Sánchez-Loyo, L., Morfín, T., García, J., Quintanilla, R., Hernández, H., Contreras, E. \& Cruz, J. (2014). Intento de suicidio en adolescentes mexicanos: perspectiva desde el consenso cultural. Acta de Investigación Psicológica, 4(1), 1446-1458. http://dx.doi.o $\mathrm{rg} / 10.1016 / \mathrm{S} 2007-4719(14) 70386-2$

Sarmiento, S. S. \& Aguilar, J. (2011). Predictores familiares y personales de la ideación suicida en adolescentes. Revista Psicología y Salud, 21 (1), 25-30. Recuperado de http://revistas.uv.mx/index.php/psicysal ud/article/view/583/1006 
Shaffer, D., Fisher, P., Dulcan, M., Davies, M., Piacentini, J., Schwab-Stone, M.,... Regier, D. A. (1996). The NIMH Diagnostic Interview Schedule for Children version 2.3 (DISC-2.3): Description, acceptability, prevalence rates and performance in the MECA study. Journal of the American Academy of Child and Adolescent Psychiatry, 35, 865-877. http://dx.doi.org/10.1097/000 04583-199607000-00012

Shaffer, D., Gould, M., Fisher, P., Trautman, P., Moreau, D., Kleinman M., \& Flory, M. (1996). Psychiatric diagnosis in child and adolescent suicide. Archives of General Psychiatry, 53(4), 339-348. http://dx.doi.or g/10.1001/archpsyc. 1996.01830040075012

Shaffer, D., Gould, M., \& Hicks, R. (1994). Worsening suicide rate in Black teenagers. American Journal of Psychiatry, 151, 18101812.

Shaffer, D., \& Greenberg, T. (2002). Suicide and suicidal behavior in children and adolescents. En D. Shaffer \& B. D. Waslick (Eds.), The many faces of depression in children and adolescents (pp. 129-159). Washington, DC: American Psychiatric Publishing.

Shaffer, D., Scott, M., Wilcox, H., Maslow, C., Hicks, R., Lucas, C., \& Greenwald, S. (2004). The Columbia Suicide Screen: validity and reliability of a screen for youth suicide and depression. Journal of the American Academy of Child and Adolescent Psychiatry, 43(1), 71-9. http://10.1097/0000 4583-200401000-00016

Shneidman, E. S. (1992). A conspectus of the suicidal scenario. En R. W. Maris, A. L. Berman, J. T. Maltsberger \& R. I. Yufit (Dirs.), Assessment and prediction of suicide (pp. 50-64). Nueva York: Guilford.

Shur-Fen Gau, S., Chen, Y., Tsai, F., Lee, M., Chiu, Y., Soong, W., \& Hwu, H. (2008). Risk factors for suicide in Taiwanese college students. Journal of American College Health, 57, 135-142. http://dx.doi.org/10.3200/JA CH.57.2.135-142

Silverman, M., \& Felner, R. (1995). Suicide prevention programs: Issues of design, implementation, feasibility, and developmental appropriateness. Suicide and Life-Threatening Behavior, 25(1), 92-104. http://dx.doi.org/10.1111/j.1943-2 78X.1995.tb00395.x

Silvikena, A., \& Kvernmob, S. (2007). Suicide attempts among indigenous Sami adolescents and majority peers in Arctic Norway: Prevalence and associated risk factors. Journal of Adolescence, 30, 613-626. http://dx.doi.org/10.1016/j.adoles cence.2006.06.004

Soloff, P., Lynch, K., Kelly, T., Malone, K., \& Mann, J. (2000). Characteristics of suicide attempts of patients with major depressive episode and borderline personality disorder: A comparative study. The American Journal of Psychiatry, 157(4), 601-608. http://dx.do i.org/10.1176/appi.ajp.157.4.601

Souminen, K., Isometsa, E., \& Lönquist, J. (2004). Level of suicidal intent predicts overall mortaly alter attempted suicide: A 12 year follow-up study. BMC Psychiatry, 4(11). http://dx.doi.org/10.1186/1471-244 $\mathrm{X}-4-11$

Urzúa, F., \& Caqueo-Urízar, A. (2011). Construcción y evaluación psicométrica de una escala para pesquisar factores vinculados al comportamiento suicida en adolescentes chilenos. Universitas Psychologica, 10(3), 721-734. Recuperado de http://revistas.javeriana.edu.co/index.p $\mathrm{hp} / \mathrm{revPsycho/article/view/564}$

Villacieros, M., Bermejo, J., Magaña, M., \& Fernández-Quijano, I. (octubre, 2016). Psychometric properties of the Attitudinal Beliefs Questionnaire about suicidal behavior (CCCS-18). The Spanish Journal of Psychology, 19, E68. http://dx.doi.org/10.10 17/sjp.2016.68

Villalobos-Galvis, F. (2009). Fiabilidad y validez del Inventario de Ideación Suicida Positiva y Negativa-PANSI, en estudiantes colombianos. Universitas Psychologica, 9(2), 509-520. Recuperado de http://revistas.javeriana.edu.co/index.p hp/revPsycho/article/viewFile/243/470 
Villalobos-Galvis, F., Arévalo Ojeda, C., \& Rojas Rivera, F. (2012). Adaptación del Inventario de Resiliencia ante el Suicidio (SRI-25) en adolescentes y jóvenes de Colombia. Revista Panamericana de Salud Pública, 31 (3), 233-239. http://dx.doi.org/1 0.1590/S1020-49892012000300008

Wolfersdorf, M., \& Kaschka, W. (Eds.). (1995). Suizidalität-Die biologische Dimension. Berlin, Heidelberg/Nueva York: Springer.

World Health Organization. (2008). Suicide rates (per 100 000) by country, year and sex. Ginebra: Autor. Recuperado de http://www .who.int/mental health/media/en/426.pdf

Zahl, D., \& Hawton, K. (2004). Repetition of deliberate self-harm and subsequent suicide risk: Long-term follow-up study of 11583 patients. The British Journal of Psychiatry, 185, 70-75. http://dx.doi.org/10.1192/bjp.1 85.1 .70

\section{Apéndice 1}

\section{Escala de evaluación del riesgo suicida ERS}

\begin{tabular}{lll}
\hline Ítem & \multicolumn{1}{c}{ Redacción } & \multicolumn{1}{c}{ Factor } \\
\hline 1 & Creo que es mejor no hacer planes para un futuro & \\
2 & Pienso que el futuro es incierto y triste & Depresión y \\
3 & Creo que nada bueno me espera & desesperanza \\
4 & Pienso que la vida no tiene cosas buenas para ofrecerme & \\
5 & Mi vida ha sido un fracaso y dudo que cambie en un futuro & \\
6 & Me siento incómodo(a) conmigo mismo(a) y con lo que he logrado & \\
7 & He pensado seriamente en quitarme la vida & \\
8 & $\begin{array}{l}\text { Pienso que la muerte es la única manera de terminar con mi } \\
\text { sufrimiento }\end{array}$ & $\begin{array}{l}\text { Ideación e } \\
\text { intencionalidad }\end{array}$ \\
9 & He planeado como quitarme la vida & suicida \\
10 & He intentado quitarme la vida alguna vez & \\
11 & He hecho cosas contra mí para no seguir viviendo & \\
12 & Me he lastimado o herido intencionalmente & Aislamiento/ \\
13 & Me siento solo(a) en la vida & \\
14 & Me es dificil confiar en otras personas & \\
15 & Me gustaría tener amigos a quienes acudir cuando tengo problemas & soporte social \\
16 & Creo que las personas tienden a ignorarme & \\
17 & A mi familia le interesa poco lo que suceda conmigo & Falta de apoyo \\
18 & Prefiero acudir a personas diferentes a mi familia cuando necesito & familiar \\
& ayuda & \\
19 & Prefiero estar lejos de mi familia que compartir con ellos & \\
20 & Pienso que soy poco importante para mi familia & \\
\hline
\end{tabular}

\section{Apéndice 2}

Puntuaciones y centiles hombres 13-18 años

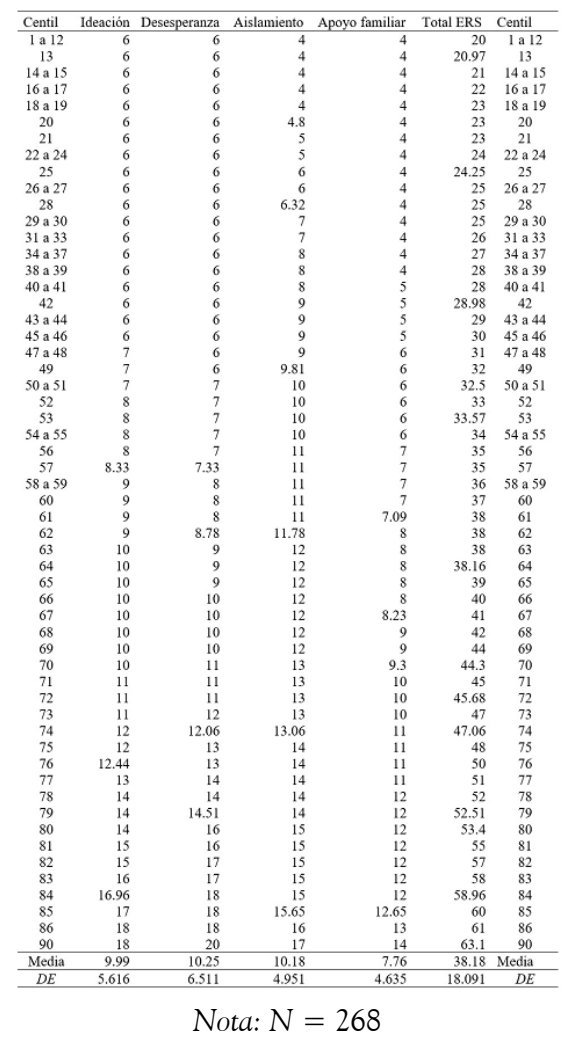




\section{Apéndice 3}

Puntuaciones y centiles mujeres $13-18$ años

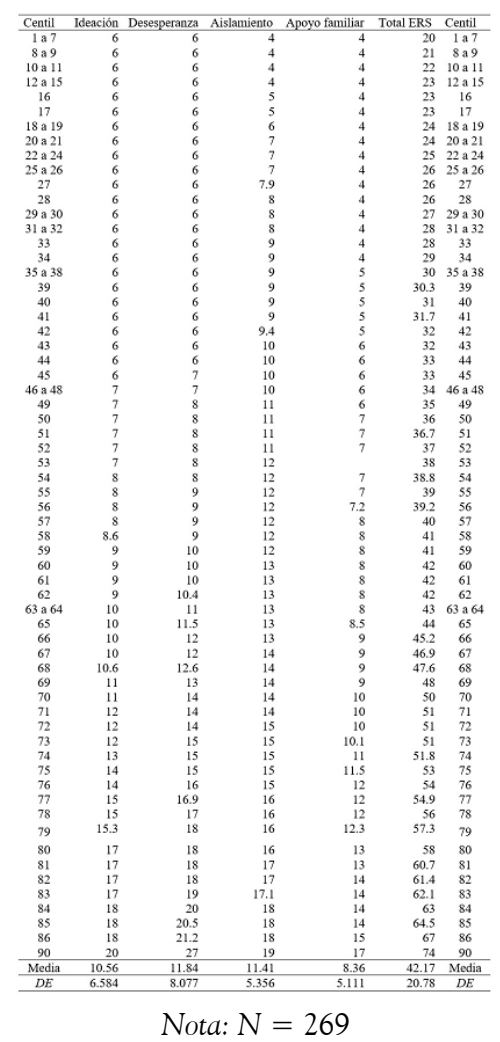

\section{Notas}

* Artículo de investigación. Esta investigación obtuvo financiación mediante convocatoria interna de la Universidad Simón Bolívar de Barranquilla. 